\title{
SPECTROSCOPIC DIAGNOSIS OF MAGNETIC FIELDS OF AP STARS
}

\author{
G. MATHYS \\ European Southern Observatory \\ Casilla 19001, Santiago 19, Chile
}

\section{Introduction}

Diagnosis of Ap star magnetic fields through spectroscopic methods is a broad topic, which cannot be fully covered within the rather tight limits of this contribution. Accordingly, the latter is devoted to only one particular approach, namely the determination of the mean magnetic field modulus from the observation of resolved magnetically split lines in high-dispersion spectra taken in unpolarized light. This determination is straightforward, mostly approximation free and model independent. The derived quantity, the mean magnetic field modulus, is the line-intensity weighted average over the visible stellar hemisphere of the modulus of the magnetic vector.

Magnetically split lines can be resolved observationally in an Ap star provided not only that the star has a strong enough magnetic field, but also that its projected equatorial velocity is low enough so that split components are not smeared out by rotational Doppler broadening. Accordingly, magnetically resolved lines are observed only in a fraction of Ap stars. By the end of the 1980's, only 12 such stars were known, and for only 4 of them, the magnetic field variations had been studied throughout the rotation cycle.

Here I present the results of a major project that has been carried out over the last years to study systematically Ap stars with resolved magnetically split lines. This project is still going on; I report about its status as of August 1995. These results are presented in greater detail elsewhere (Mathys et al. 1997). In this contribution, I only summarize them briefly.

\section{Observations and analysis}

All the determinations of the mean magnetic field modulus within the framework of the current project rely on the measurement of the wavelength shift between the magnetically split components of the line Fe II $\lambda 6149.2$, in high-resolution spectra recorded in unpolarized light. The line Fe II $\lambda 6149.2$ has a particularly simple Zeeman pattern: it is a doublet, that is, it shows only two magnetically split components. The separation of these two components is unusually large (the Landé factor is 2.7), which together with the simplicity of the pattern makes the line especially appropriate for magnetic field diagnosis.

For this project, high-resolution spectra of the region around Fe II $\lambda 6149.2$ have been recorded for a large sample of Ap stars. The purpose was twofold: to carry out a systematic search for Ap stars with resolved magnetically split lines, and to observe regularly such stars already known so as to study the variation of their magnetic field throughout their rotation cycle.

The observations were carried out with a variety of telescope and instrument combinations, at the following observatories: European Southern Observatory, Observatoire de Haute-Provence, Kitt Peak National Observatory, and Canada-France-Hawaii Telescope. In total, 222 nights of observation had been fully or partly devoted to this project until August 1995.

\section{Results}

Within the course of this project, 29 Ap stars with resolved magnetically split lines have been discovered. This raises the number of such stars presently known to 42 , compared to 12 when this study was started (the 42 nd star with magnetically resolved lines has been discovered more recently 
by Babel et al. 1995). As of end of August 1995, more than 750 measurements of the mean magnetic field modulus of 40 of those 42 stars had been obtained (compared to about 80 measurements in the literature at the time of the beginning of this investigation). Those measurements, in the most favourable cases (more than one third of the studied stars), reach an unprecedented accuracy (25$30 \mathrm{G}$ ). This study brought the number of stars with field modulus measurements sampling (fairly) well the rotation cycle from 4 to 16 . For a few more stars good phase coverage should soon be achieved.

These achievements allow one to discuss various aspects of the magnetism of Ap stars in greater generality and on sounder statistical grounds than has previously been possible. The main conclusions are summarized below.

The most intriguing outcome of this study is the discovery of a discontinuity at the low end of the distribution of the mean magnetic field moduli of Ap stars. No star with a field modulus (averaged over the rotation cycle) lower than $2.8 \mathrm{kG}$ has been found, although the resolution limit was close to $1.7 \mathrm{kG}$. Yet, a number of stars with very sharp, unresolved lines have been observed. It is not clear if these stars have magnetic fields much weaker than $1.7 \mathrm{kG}$ or no field at all, hence if the discontinuity in the distribution is a gap or a cutoff. One of my goals for the future is to try to answer this question, by pushing down the limit of field detection in the sharpest-lined Ap stars. Possible approaches for this are:

- to look for magnetically resolved split lines in high-resolution infrared spectra (to take advantage of the fact that the Zeeman effect increases quadratically with wavelength). Instruments suited to that purpose are just coming on line, like the Phoenix spectrograph at KPNO;

- to diagnose the mean quadratic magnetic field of those stars by application of the moment technique to large samples of lines in high-resolution cross-dispersed echelle spectra covering a broad wavelength range. A first exploration of this approach has recently been carried out by Mathys \& Hubrig (1997).

Both approaches appear to have the potential to lower the field detection limit by a factor of about 3 with respect to the present study.

While the lower limit of the observed magnetic field moduli seems fairly independent of the temperature, it appears that stronger fields can only be found in hotter stars. There is also some kind of anticorrelation between magnetic field modulus and stellar rotation period, in the following sense. While the field moduli measured in stars with rotation periods longer than 150 days never exceed $7.5 \mathrm{kG}$, more than half of the stars with shorter periods have fields larger than that value.

For more than one third of the studied stars, enough field determinations well distributed throughout the stellar rotation cycle have been achieved to allow the variations of the field modulus to be characterized at least to some extent. These variations are often significantly anharmonic, and it is not unusual for their extrema not to coincide in phase with the extrema of the longitudinal field (for the few stars for which enough data exist about the latter). This, together with considerations on the distribution of the relative amplitude of variation of the studied stars, supports the recently emerging evidence for markedly non-dipolar geometry and fine structure of the magnetic fields of most Ap stars. A program aimed at measuring the longitudinal fields of the stars with magnetically resolved lines is now under way: those measurements, when combined with the mean field modulus data discussed here, will allow one to derive much better constraints about the field geometry.

New or improved determinations of the rotation periods of 9 Ap stars have been achieved from the analysis of the variations of their mean magnetic field modulus. Tentative values of the period have been derived for 5 additional stars, and lower limits have been established for 10 stars. The shortest definite rotation period of an Ap star with magnetically resolved lines is 3 d, while those stars that rotate slowest appear to have periods in excess of 70 or 75 years. As a result of this study, the number of known Ap stars with rotation periods longer than 30 days is almost doubled. This provides improved knowledge of the slow-rotation tail of the period distribution of Ap stars. The distribution that is found is consistent with an equipartition of the long periods (say, over 1 year) of Ap stars on a logarithmic period scale, or in other words, with a distribution decreasing exponentially with the period.

As a by-product, this study also yielded the discovery of radial velocity variations in 8 stars. Enough data have been obtained to derive at least preliminary constraints on the orbital periods of those binaries. Combining those results with information from the literature, there seems to be a deficiency of binaries with short orbital periods among Ap stars with magnetically resolved lines. 


\section{Conclusion}

The study reported here represents a major breakthrough in our knowledge of the magnetic fields (and of some other properties) of the Ap stars. In this contribution, I have in purpose restricted myself to the presentation of the observational data, and I have not attempted to discuss their interpretation in terms of the understanding of the origin and evolution of the magnetic Ap stars. The latter is obviously the ultimate goal to achieve. This study is an important step towards that achievement. It calls for additional observational data on a number of aspects, which have been mentioned in the text above. Work is in progress to gather this complement of observations.

\section{References}

Babel J., North P., Queloz D., 1995, A\&A 303, L5

Mathys G., Hubrig S., 1997 (in preparation)

Mathys G., Hubrig S., Landstreet J.D., Lanz T., Manfroid J., 1997, A\&AS 314, 491 